\title{
Avaliação do coeficiente de variação como medida da precisão em experimentos com frangos de corte
}

\author{
[Evaluation of the coefficient of variation as a precision measure in experiments with broilers] \\ D.F. Mohallem ${ }^{1}$, M. Tavares $^{2}$, P.L. Silva ${ }^{3 *}$, E.C. Guimarães ${ }^{2}$, R.F. Freitas ${ }^{1}$ \\ ${ }^{1}$ Aluno de pós-graduação - UFU - Uberlândia, MG
${ }^{2}$ Faculdade de Matemática - UFU - Uberlândia, MG \\ ${ }^{3}$ Faculdade de Medicina Veterinária - UFU \\ Av. Pará, 1720 - Umuarama \\ 38400-902 - Uberlândia, MG
}

\begin{abstract}
RESUMO
Estabeleceram-se faixas de coeficientes de variação (CV) que orientem a avaliação de determinadas variáveis em pesquisas futuras. Foram coletados dados de CV de vários trabalhos publicados no Brasil, com ênfase em frangos de corte, abordando: ganho de peso, consumo de ração, conversão alimentar, mortalidade, viabilidade, peso vivo e rendimentos de carcaça, de peito, de pernas, de gordura abdominal, de asas e de dorso. Utilizou-se uma tabela que relaciona mediana (Md) e pseudo-sigma (PS) para estabelecer os intervalos de CV de cada variável. O rendimento de gordura abdominal apresentou faixa de variação com maiores coeficientes de variação; para as demais variáveis, as faixas de classificação dos coeficientes de variação mostraram-se relativamente baixas.
\end{abstract}

Palavras-chave: frango de corte, estatística, coeficiente de variação, precisão, avicultura

\begin{abstract}
Coefficients of variation (CV) were taken from several researches published in Brazil, with emphasis on broilers performance and their most studied characteristics, such as weight gain, feed consumption, feed:weight gain ratio, mortality, viability, body weight and yields of carcass, breast, legs, abdominal fat, wings and back. The purpose of the study was to establish $C V$ bands which can guide the evaluation of each variable in future experiments. Based on relations between the median (Md) and pseudosigma (PS), a table was created with suggested intervals for the cited parameters. Larger coefficient of variation intervals were observed for abdominal fat yield. The other variables showed relatively low $C V$ interval bands.
\end{abstract}

Keywords: broiler, statistic, coefficient of variation, precision, poultry

\section{INTRODUÇ̃̃O}

O coeficiente de variação é uma medida de dispersão empregada para estimar a precisão de experimentos e representa o desvio-padrão expresso como porcentagem da média.

Como medida de dispersão, a principal qualidade do $\mathrm{CV}$ é a capacidade de comparar resultados de diferentes trabalhos que envolvem a mesma

Recebido em 26 de fevereiro de 2007

Aceito em 31 de janeiro de 2008

*Autor para correspondência (corresponding author)

E-mail: plsilva@umuarama.ufu.br variável-resposta, permitindo quantificar a precisão das pesquisas (Kalil, 1977; Garcia, 1989). O número de repetições, tamanho das parcelas, delineamento experimental, heterogeneidade do ambiente e a diversidade genética dos animais influem no erro experimental. Em igualdade de condições, é mais preciso o experimento com menor coeficiente de variação (Garcia, 1989). De acordo com Snedecor e Cochran (1980), a distribuição do $\mathrm{CV}$ possibilita estabelecer faixas de valores que orientem os pesquisadores sobre a validade de seus experimentos. 
Na pesquisa avícola, não há referencial de valores de CV que identifique faixas de classificação quanto a seu grau de precisão, a exemplo do que ocorre com dados agronômicos (Amaral et al., 1997; Costa et al., 2002; Lima et al., 2004) e mesmo na suinocultura (Judice et al., 1999). Gomes (1991) sugere o cálculo do índice de variação, que é a relação entre o $\mathrm{CV}$ e a raiz quadrada do número de repetições, entretanto este deve ser determinado individualmente para cada trabalho e depois ser comparado.

Assim, fica evidente a necessidade de se estudar a distribuição dos valores de $\mathrm{CV}$ em pesquisas com aves, pois a maioria dos pesquisadores tem comparado os seus resultados com aqueles sugeridos por Gomes (1990). Essa classificação considera os coeficientes de variação como baixos quando inferiores a $10 \%$, médios entre 10 e $20 \%$, altos entre 20 e $30 \%$ e muito altos se superiores a $30 \%$; valores esses obtidos em experimentos de campo com culturas agrícolas e que, consequentemente, não devem ser aplicados à avicultura em que as condições experimentais são diferentes.

Amaral et al. (1997) e Judice et al. (1999) sugerem verificar a normalidade da distribuição dos coeficientes de variação para encontrar as faixas de variabilidade. Entretanto, Costa et al. (2002), ao trabalharem com dados da cultura do arroz de terras altas, apresentaram um novo método de classificação dos coeficientes de variação que pode ser aplicado independentemente da distribuição dos valores de CV. Este método baseou-se no uso da mediana (Md) e do pseudo-sigma (PS), medidas, segundo Costa et al. (2002), mais resistentes que a média e o desvio-padrão.

Quando os dados não têm distribuição normal, o uso do pseudo-sigma como uma medida de dispersão será mais resistente que o desviopadrão $(s)$ clássico. Se os dados têm distribuição aproximadamente normal, o pseudo-sigma produz uma estimativa próxima de $s$, que é o desvio-padrão da amostra (Hoaglin et al., 1983; Blanxart et al., 1992).

O objetivo deste trabalho foi avaliar a distribuição do $\mathrm{CV}$ em experimentos com frangos de corte, considerando suas principais variáveis-resposta, para estabelecer faixas de classificação que orientem o pesquisador com relação à precisão dos dados de sua pesquisa.

\section{MATERIAL E MÉTODOS}

Foram tabulados coeficientes de variação de diversos trabalhos sobre frangos de corte publicados no Arquivo Brasileiro de Medicina Veterinária (1999 a 2006), Revista Brasileira de Ciência Avícola (2000 a 2005) e Revista Brasileira de Zootecnia (2000 a 2006). Selecionaram-se as variáveis que ocorreram com maior freqüência nos artigos, quais sejam: ganho de peso, consumo de ração, conversão alimentar, mortalidade, viabilidade, peso vivo, rendimento de carcaça, rendimento de peito, rendimento de pernas (coxas e sobrecoxas), rendimento de gordura abdominal, rendimento de asas e rendimento de dorso.

Neste trabalho, não foram especificados os delineamentos experimentais, considerando a conclusão de Estefanel et al. (1987), segundo a qual tais aspectos não influenciaram significativamente os valores de $\mathrm{CV}$, pressupondo-se que a forma de disposição da pesquisa visa, em princípio, atenuar a possibilidade do erro experimental.

Para testar o ajuste dos dados à distribuição normal, utilizou-se o método de KolmogorovSmirnov, modificado por Lilliefors (1967).

Para cada variável-resposta, foram obtidos: maior valor, menor valor, amplitude, média, mediana, primeiro quartil, terceiro quartil, amplitude interquartílica e pseudo-sigma.

As faixas de classificação foram definidas de acordo com o modelo proposto por Costa et al. (2002), que consideraram: baixo: $\mathrm{CV} \leq$ (Md - PS), médio: $(\mathrm{Md}-\mathrm{PS})<\mathrm{CV} \leq(\mathrm{Md}+\mathrm{PS})$, alto: $(\mathrm{Md}+$ $\mathrm{PS})<\mathrm{CV} \leq$ (Md + 2PS), muito alto: $\mathrm{CV}>(\mathrm{Md}+$ 2PS $)$, em que: $\mathrm{Md}=\left(\mathrm{Q}_{1}+\mathrm{Q}_{3}\right) / 2$ é a mediana dos coeficientes de variação, $Q_{1}$ e $Q_{3}$ são o primeiro e terceiro quartis, respectivamente, os quais delimitam $25 \%$ de cada extremidade da distribuição e $\mathrm{PS}=\mathrm{IQR} / 1,35$ é o pseudo-sigma sendo IQR, amplitude interquartílica $\left(\mathrm{IQR}=\mathrm{Q}_{3}-\mathrm{Q}_{1}\right)$, medida resistente que indica o quanto os dados estão distanciados da mediana.

Sob a condição de normalidade, tem-se que $68,3 \%$ dos CV estão incluídos entre Md $\pm 1 \mathrm{PS} ; 95,4 \%$ dos CV estão entre $\mathrm{Md} \pm 2 \mathrm{PS}$; e 99,7\% dos CV estão entre Md \pm 3 PS (Spiegel, 1993). Portanto, as freqüências esperadas dos coeficientes de variação nas faixas de classificação definidas acima correspondem a: $15,9 \%, 68,3 \%, 13,6 \%$ e $2,3 \%$, respectivamente. 


\section{RESULTADOS E DISCUSSÃO}

A aplicação do teste de normalidade de Lilliefors mostrou que somente as variáveis viabilidade, rendimento de gordura abdominal, rendimento de asas e rendimento de dorso tiveram distribuição normal com 5\% de significância.

Nas Tab. 1 e 2, são apresentados os resultados estatísticos obtidos a partir dos valores de CV encontrados na literatura.

A variável mortalidade apresentou elevada variação em seus CV, resultando em pseudo-sigma de $86,7 \%$ e amplitude de 453,2\% (Tab. 1 e 2). Esses altos valores devem-se à própria natureza da variável, que por ter um valor muito baixo sofre, proporcionalmente, grandes alterações com pequenas mudanças absolutas. Os valores encontrados para Md e PS de mortalidade tornaram a faixa de classificação dos $\mathrm{CV}$ incoerente, não sendo, portanto, estipulada. Por não ser uma variável com boa precisão (grande amplitude de CVs), seu uso não é aconselhável. Em seu lugar, é indicada a viabilidade que possui características mais estáveis, mostrando PS $1,4 \%$ e amplitude de $8,3 \%$, induzindo a menores chances de erro experimental.

Tabela 1. Teste de normalidade de Lilliefors e estatística descritiva dos coeficientes de variação das variáveis estudadas nos experimentos publicados sobre frangos de corte

\begin{tabular}{lcccc}
\hline \multicolumn{1}{c}{ Variável } & Lilliefors & $\begin{array}{c}\text { Maior valor } \\
(\%)\end{array}$ & $\begin{array}{c}\text { Menor Valor } \\
(\%)\end{array}$ & $\begin{array}{c}\text { Amplitude } \\
(\%)\end{array}$ \\
\hline Ganho de peso & $0,0000^{*}$ & 30,30 & 0,62 & 29,68 \\
Consumo de ração & $0,0000^{*}$ & 18,24 & 0,34 & 17,90 \\
Conversão alimentar & $0,0000^{*}$ & 29,04 & 0,73 & 28,31 \\
Mortalidade & $0,0000^{*}$ & 453,17 & 0,00 & 453,17 \\
Viabilidade & $0,0607^{\text {ns }}$ & 9,16 & 0,86 & 8,30 \\
Peso vivo & $0,0011^{*}$ & 8,65 & 0,67 & 7,98 \\
Rendimento de carcaça & $0,0000^{*}$ & 9,48 & 0,80 & 8,68 \\
Rendimento de peito & $0,0208^{*}$ & 10,96 & 1,43 & 9,53 \\
Rendimento de pernas & $0,0196^{*}$ & 6,40 & 1,64 & 4,76 \\
Rendimento de gordura & $0,2000^{\text {ns }}$ & 34,60 & 6,45 & 28,15 \\
abdominal & $0,0536^{\text {ns }}$ & 11,65 & 1,65 & 10,00 \\
Rendimento de asas & $0,0790^{\text {ns }}$ & 11,37 & 1,50 & 9,87 \\
Rendimento de dorso & &
\end{tabular}

* significativo em nível de 0,05 no teste de Lilliefors.

${ }^{\text {ns }}$ não significativo em nível de 0,05 no teste de Lilliefors.

Tabela 2. Variáveis mais utilizadas em experimentos com frangos de corte e suas respectivas estimativas da média, pseudo-sigma e mediana dos coeficientes de variação, em porcentagem, e número de experimentos

\begin{tabular}{lcccc}
\hline Variável & $\mathrm{N}^{1}$ & Média & Pseudo $\sigma$ & Mediana \\
\hline Ganho de peso & 277 & 4,65 & 1,96 & 3,54 \\
Consumo de ração & 288 & 3,98 & 1,49 & 3,45 \\
Conversão alimentar & 294 & 4,15 & 1,98 & 3,29 \\
Mortalidade & 44 & 94,35 & 86,73 & 42,83 \\
Viabilidade & 30 & 3,22 & 1,43 & 2,70 \\
Peso vivo & 76 & 3,29 & 1,60 & 2,89 \\
Rendimento de carcaça & 83 & 2,16 & 0,83 & 1,86 \\
Rendimento de peito & 73 & 4,28 & 1,95 & 3,98 \\
Rendimento de pernas & 53 & 3,27 & 1,39 & 2,94 \\
Rendimento de gordura & 63 & 16,84 & 5,83 & 15,99 \\
abdominal & 26 & 4,39 & 2,10 & 3,77 \\
Rendimento de asas & 25 & 4,65 & 1,59 & 4,30 \\
Rendimento de dorso & & & & \\
\hline
\end{tabular}

${ }^{1}$ número de experimentos 
Ganho de peso e conversão alimentar apresentaram amplitude de $29,7 \%$ e $28,3 \%$ (Tab. $1)$, respectivamente, sendo esse elevado valor atribuível à grande diversidade de pesquisas, uma vez que não foram especificados os delineamentos experimentais. Para ganho de peso, a média dos $\mathrm{CV}$ foi de $4,6 \%$ e, para conversão alimentar, de $4,15 \%$, evidenciando que a ocorrência de coeficientes de variação altos para essas características não são comuns.

Como demonstrado por sua média de CV (Tab. 2), o rendimento de gordura abdominal é uma variável com comportamento diferente das demais. Exceção feita à mortalidade, por suas características já comentadas, o rendimento de gordura abdominal é a característica que apresentou o maior valor de CV máximo assim como o maior valor de CV mínimo (Tab. 1).

Analisando-se a metodologia proposta por Costa et al. (2002), a Tab. 3 deve ser utilizada como referência pelo pesquisador para verificar se os resultados de CV obtidos estão, ou não, dentro de uma faixa esperada de valores.

Tabela 3. Faixas de classificação para os coeficientes de variação (\%) das variáveis comumente avaliadas em experimentos com frangos de corte

\begin{tabular}{lcccc}
\hline Variável & Baixo & Médio & Alto & Muito alto \\
\hline Ganho de peso & $\mathrm{CV} \leq 1,577$ & $1,577<\mathrm{CV} \leq 5,503$ & $5,503<\mathrm{CV} \leq 7,466$ & $\mathrm{CV}>7,466$ \\
Consumo de ração & $\mathrm{CV} \leq 1,956$ & $1,956<\mathrm{CV} \leq 4,937$ & $4,937<\mathrm{CV} \leq 6,428$ & $\mathrm{CV}>6,428$ \\
Conversão alimentar & $\mathrm{CV} \leq 1,306$ & $1,306<\mathrm{CV} \leq 5,269$ & $5,269<\mathrm{CV} \leq 7,250$ & $\mathrm{CV}>7,250$ \\
Mortalidade & - & - & - & - \\
Viabilidade & $\mathrm{CV} \leq 1,269$ & $1,269<\mathrm{CV} \leq 4,121$ & $4,121<\mathrm{CV} \leq 5,547$ & $\mathrm{CV}>5,547$ \\
Peso vivo & $\mathrm{CV} \leq 1,285$ & $1,285<\mathrm{CV} \leq 4,485$ & $4,485<\mathrm{CV} \leq 6,085$ & $\mathrm{CV}>6,085$ \\
Rendimento de carcaça & $\mathrm{CV} \leq 1,034$ & $1,034<\mathrm{CV} \leq 2,686$ & $2,686<\mathrm{CV} \leq 3,512$ & $\mathrm{CV}>3,512$ \\
Rendimento de peito & $\mathrm{CV} \leq 2,032$ & $2,032<\mathrm{CV} \leq 5,928$ & $5,928<\mathrm{CV} \leq 7,876$ & $\mathrm{CV}>7,876$ \\
Rendimento de pernas & $\mathrm{CV} \leq 1,547$ & $1,547<\mathrm{CV} \leq 4,333$ & $4,333<\mathrm{CV} \leq 5,725$ & $\mathrm{CV}>5,725$ \\
Rendimento de gordura & $\mathrm{CV} \leq 10,160$ & $10,160<\mathrm{CV} \leq 21,820$ & $21,820<\mathrm{CV} \leq 27,649$ & $\mathrm{CV}>27,649$ \\
abdominal & & & & \\
Rendimento de asas & $\mathrm{CV} \leq 1,666$ & $1,666<\mathrm{CV} \leq 5,874$ & $5,874<\mathrm{CV} \leq 7,977$ & $\mathrm{CV}>7,977$ \\
Rendimento de dorso & $\mathrm{CV} \leq 2,707$ & $2,707<\mathrm{CV} \leq 5,893$ & $5,893<\mathrm{CV} \leq 7,485$ & $\mathrm{CV}>7,485$ \\
\hline
\end{tabular}

Ao se compararem as faixas de classificação encontradas (Tab. 3) com a proposta por Gomes (1990), na qual valores de CV menores que 10 são considerados baixos, fica evidente a disparidade das informações. $\mathrm{O}$ rendimento de carcaça destaca-se como característica com menor mediana de CV (Tab. 2), o que reflete em sua faixa de classificação bastante estreita (Tab. 3). O rendimento de gordura abdominal teve uma faixa classificação de CV próxima ao preconizado por Gomes em (1990). Excetuandose o rendimento de gordura abdominal, para as demais variáveis, um CV de $8,0 \%$ é considerado muito alto, diferentemente do preconizado pelo referido autor.

Gomes (1990) estipulou as faixas de classificação de CV com base em variáveis agronômicas, entretanto, na avicultura de corte, as variações não controladas, ou frutos do acaso, são relativamente menores que as encontradas nos experimentos agronômicos. Isso porque se trabalha com linhagens e híbridos, dentro de um ambiente com certo grau de controle, esperando alta homogeneidade. Portanto, essas faixas estipuladas por Gomes (1990), apesar de serem amplamente utilizadas como valores referenciais, induzem a um grande viés nas conclusões dos experimentos na área de avicultura de corte.

\section{CONCLUSÕES}

As faixas de classificação dos coeficientes de variação encontradas para as variáveis analisadas podem ser utilizadas como referência para determinar a precisão experimental. Os coeficientes de variação em estudos com frangos de corte têm características próprias e diferem significativamente das faixas propostas por Gomes (1990) para a maioria das variáveis analisadas. As faixas de classificação dos coeficientes de variação mostraram-se relativamente baixas, indicando homogeneidade das condições experimentais. 


\section{REFERÊNCIAS BIBLIOGRÁFICAS}

AMARAL, A.M.; MUNIZ, J.A.; SOUZA, M. Avaliação do coeficiente de variação como medida da precisão na experimentação com citros. Pesq. Agropecu. Bras., v.32, p.1221-1225, 1997.

BLANXART, M.F.; COSIALLS, L.S.; OLMOS, J.G. et al. Análisis exploratorio de datos: nuevas técnicas estadísticas. Barcelona: Promociones y Publicaciones Universitarias, 1992. 296p.

COSTA, N.H.A.D.; SERAPHIN, J.C.; ZIMMERMANN, F.J.P. Novo método de classificação de coeficientes de variação para a cultura do arroz de terras altas. Pesq. Agropecu. Bras., v.37, p.243-249, 2002.

ESTEFANEL， V.; PIGNATARO, I.A.B.; STORCK, L. Avaliação do coeficiente de variação de experimentos com algumas culturas agrícolas. In: SIMPÓSIO DE ESTATÍSTICA APLICADA À EXPERIMENTAÇÃO AGRONÔMICA, 2., 1987, Londrina. Anais... Londrina: UEL/RBRAS, 1987. p.115-131.

GARCIA, C.H. Tabelas para classificação do coeficiente de variação. Piracicaba: IPEF, 1989. 12p. (Circular técnica, 171).

GOMES, F.P. Curso de estatística experimental. 12.ed. São Paulo: Nobel, 1990. 467p.
GOMES, F.P. $O$ indice de variação, um substituto vantajoso do coeficiente de variação. Piracicaba: IPEF, 1991. 4p. (Circular técnica, 178).

HOAGLIN, D.C.; MOSTELLER, F.; TUCKEY, J.W. Understanding robust and exploratory data analysis. New York: J. Wiley, 1983. 477p.

JUDICE, M.G.; MUNIZ, J.A.; CARVALHEIRO, R. Avaliação do coeficiente de variação na experimentação com suínos. Cienc. Agrotec., v.23, p.170-173, 1999.

KALIL, E.B. Princípios de técnica experimental com animais. Piracicaba: ESALQ/USP, 1977. 210p.

LILLIEFORS, H.W. On the KolmogorovSmirnov test for normality with mean and variance unknown. J. Am. Stat. Assoc., v.62, p.399-402, 1967.

LIMA, L.L.; NUNES, G.H.S.; BEZERRA NETO, F. Coefficients of variation of some melon yield components and fruit quality traits: a proposal for classification. Hortic. Bras., v.22, p.14-17, 2004.

SNEDECOR, G.W.; COCHRAN, W.G. Statistical methods. 7.ed. Ames: The Iowa State University, 1980. 593p.

SPIEGEL, M.R. Estatística. 3.ed. São Paulo: Makron Books, 1993. 643p. (Coleção Schaum). 